\title{
Simulation results, Post-processing experimentations and Comparisons results for navigation, homing and multiple vehicles operations with a new Positioning method using on transponder
}

\author{
P. Baccou \\ "Datacep engineering" \\ 78 avenue du général Leclerc \\ 92340 Bourg la reine, France \\ pbaccou@hotmail.com
}

\begin{abstract}
Homing and navigation capabilities are essential for many Autonomous Underwater Vehicle (AUV) applications. This paper presents the both problems with respect to a single beacon and an extension of the method for multiple AUV operation using relative location. The difficulties of this approach are due to the fact that a single range measurement does not completely constrain the beacon's position in the vehicle frame. In order to triangulate his position, the AUV need to maneuver while measuring its displacements between ranges. In addition, range measurements are noisy and sometimes spurious, speed bias and underwater currents affect dead-reckoning measurements. All operation need to have the same beginning. In a first time, we have an initialization phase which is necessary to obtain an initial estimate of the vehicle's location with respect to a fixed or moving beacon. These initials estimates are refined during the actual displacement towards the beacon by means of a Kalman filter. Two kinds of navigation are used so as to maximize the information matrix and to maintain an accurate absolute position. We present also postprocessing results in comparison with LBL navigation and mission experimentation post-processing treatment. And then we applied our method for two vehicles operations.
\end{abstract}

\section{INTRODUCTION}

Actually, there is a real challenge on application of Autonomous Underwater Vehicle, particularly for offshore industry, marine laboratory or military operation in bathymetry exploration, pipeline verification, environmental measurements or minefield detection. Instead of realizing many missions with one AUV, the future is to plan one mission with a flotilla.

In some cases, more sophisticated capabilities such as homing and docking are required. For the long term deployment of AUVs (Autonomous Ocean Sampling Network concept), the vehicles must be capable of returning to their moorings for recharging battery, data downloading and mission programming. Many different approaches have then been implemented and tested based on acoustic (Ultra Short Base Line, Short Base Line and Long Base Line system), electromagnetic or optical sensors.

\author{
B. Jouvencel \\ Laboratoire d'Informatique, de Robotique \\ et de Microélectronique de Montpellier \\ (LIRMM, Université Montpellier II - UM C 5560) \\ 161 rue Ada, 34392 Montpellier CX 5, France \\ jouvence@lirmm.fr
}

Long baseline navigation systems have generally been used for positioning of underwater equipment and manned submersibles, and more recently for navigation of Autonomous underwater vehicles [1][2][3][4]. Although, these systems can offer a good positioning accuracy, provided the array is correctly calibrated, they also have several drawbacks. The deployment and the recovery of the transponders as well as the calibration of the array are ship-time consuming and therefore expensive. Furthermore, the whole process has to be repeated each time the array is moved to a different place. The overall cost of the system is also substantial, given the cost of each beacon.

A different approach for AUV positioning and navigation consists of using a single beacon [5][6][7][8]. The advantage of such a solution is that calibration is reduced to the determination of the beacon's location. However, it is not possible to determine the vehicle's position from a single ping. In this case, the baselines between ranges are then created by the AUV displacements, which have to be measured with a maximal accuracy.

Contrary to the solution presented in [7], which relies on an accurate dead reckoning system to measure the displacements, our concern is to provide a solution for a vehicle equipped with low-cost sensors (follower vehicle). So, we consider that a Doppler velocity log is too expensive to be integrated on our vehicles. We rather consider that the vehicle's speed is approximately known by an priori calibration of the vehicle's water speed as a function of propeller rpm.

The paper is organized as follows: section II defines important reference frame. Section III describes the initialization procedure allowing to obtain the initial state estimate and its error covariance matrix used to initialize the Kalman filter. Section IV describes the Kalman filter in details and particularly how the vehicle's motion between ping and reply is taken into account. Section V describes different heading command for homing or navigation thanks to improve the information matrix. In section VI, we explain the extension for Multiple vehicle operation. Section VII 
presents simulation results. In section VIII, we compare our algorithm with LBL navigation. We show in section IX real post-processing experimentation with our vehicle, TAIPAN. For the end, we expose some multiple vehicle navigation in section $\mathrm{X}$.

\section{DEFINITIONS}

The location of the beacon in the local absolute reference frame $\mathrm{R}_{0}$ is $\left(\mathrm{x}_{\mathrm{b}}, \mathrm{y}_{\mathrm{b}}, \mathrm{z}_{\mathrm{b}}\right)$. This location can be known or not by the AUV, it depends on the mission, if we work in local reference, we use $(0,0, \nsucceq)$ location. The axes $\mathrm{x}_{0}$ and $\mathrm{y}_{0}$ respectively point north and east, and $\mathrm{z}_{0}$ points down. The mobile frame $R_{m}$ is located at the center of gravity of the vehicle with its axes $x_{m}, y_{m}, z_{m}$ parallel to $\mathrm{x}_{0}, \mathrm{y}_{0}, \mathrm{z}_{0}$. The knowledge of the beacon's depth allows to convert the $3 \mathrm{D}$ problem into a $2 \mathrm{D}$ problem. The terminology 'range' in the remaining of the paper should then be understood as $\mathrm{x}-\mathrm{y}$ range (slant range corrected for the depth difference between the vehicle and the beacon). The acoustic travel times are measured like in a Long Base Line system: the vehicle pings, the beacon replies upon reception after a given timeout and the vehicle detects the time of arrival of the reply. Slant ranges are calculated based on the known average speed of sound.

\section{INITIALIZATION}

\section{A. Principle}

The initialization of the beacon's location consists of commanding a $360^{\circ}$ rotation to the AUV while ranging to the beacon and measuring the displacements between pings (Fig.1). The vehicle would thus describe a circular path in the absence of underwater current or a distorted circle otherwise. In the presence of a speed bias (du) and of an underwater current $\left(\mathrm{v}_{\mathrm{cn}}, \mathrm{v}_{\mathrm{ce}}\right)$ all assumed to be constant, the north and east components of the vehicle's displacement over a sampling period $\Delta \mathrm{t}$ can be modeled by:

$$
\left\{\begin{array}{l}
\Delta x=C \theta C \psi(u-d u) \Delta t+v_{c n} \Delta t \\
\Delta y=C \theta S \psi(u-d u) \Delta t+v_{c e} \Delta t
\end{array}\right.
$$

Where $u$ is the vehicle's calibrated water referenced speed, $\theta$ is the pitch and $\psi$ is the heading. The displacements between ranges $i$ and $n$ can then be written:

$$
\left\{\begin{array}{l}
\Delta x_{i, n}=\sum_{\substack{k=l \\
k=n}}^{k=n} C \theta_{k} C \psi_{k} u_{k} \Delta t-d u \sum_{\substack{k=1 \\
k=n}}^{k=n} C \theta_{k} C \psi_{k} \Delta t+v_{c n} \sum_{\substack{k=i \\
k=n}}^{k=n} \Delta t \\
\Delta y_{i, n}=\sum_{k=i}^{k=n} C \theta_{k} S \psi_{k} u_{k} \Delta t-d u \sum_{k=1}^{n=1} C \theta_{k} S \psi_{k} \Delta t+v_{c} \sum_{k=i} \Delta t
\end{array}\right.
$$

Let $R_{m}$ be the mobile frame at the time corresponding to the end of the rotation with the measure $d_{n}$ and $(x, y)$ be the vehicle's absolute position at that time.

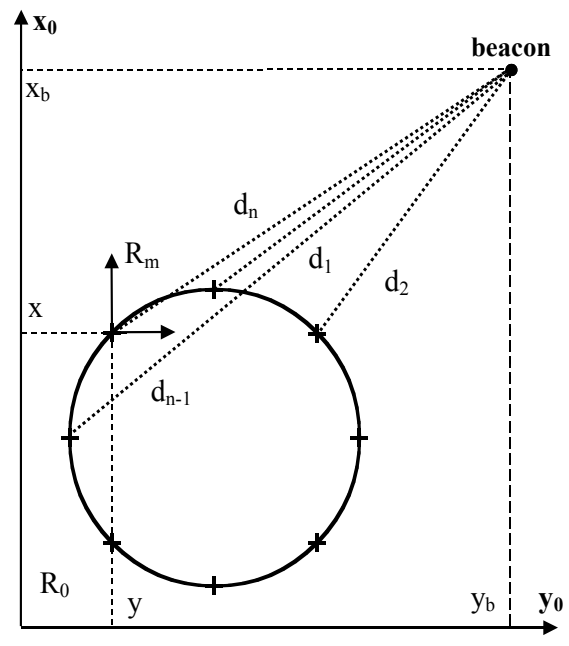

Fig.1: Geometry of the initialization procedure

The $i^{\text {th }}$ range can be expressed as a function of the vehicle's position at the end of the rotation and of the displacement between ranges $\mathrm{i}$ and $\mathrm{n}$ by:

$$
\mathrm{d}_{i}=\left[\left(\mathrm{xb}-\mathrm{x}+\Delta \mathrm{x}_{\mathrm{i}, \mathrm{n}}\right)^{2}+(\mathrm{yb}-\mathrm{y}+\Delta \mathrm{yi}, \mathrm{n})^{2}\right]^{1}
$$

The system of equations (3) can be solved for $\left(\mathrm{x}, \mathrm{y}, \mathrm{du}, \mathrm{v}_{\mathrm{cn}}, \mathrm{v}_{\mathrm{ce}}\right)$ by non-linear least squares using the Levenberg-Marquardt algorithm. However, because the problem can have local minima, we could not initialize the search with $(0,0,0,0,0)$ without risking convergence to an erroneous solution.

Therefore, in order to initialize the algorithm closer to the correct solution, the system without current and speed bias is first solved starting at $(x, y)=(0,0)$. The approximate vehicle position obtained in that case is then used to initiate the search in the presence of perturbations (the current components and the bias being still initialized at 0 ).

\section{B. Range pre-processing}

In our simulations, we chose to use a selection of 14 ranges for the estimation of $\left(\mathrm{x}, \mathrm{y}, \mathrm{du}, \mathrm{v}_{\mathrm{cn}}, \mathrm{v}_{\mathrm{ce}}\right)$. Since ranges can sometimes be spurious because of noise or multi path, the 14 ranges have to be selected carefully. The set of ranges is first pre-filtered as follows: the difference between successive ranges is computed and a threshold is applied. If the difference is greater than the threshold, further testing is performed by looking at the ranges before and after the considered pair. Depending on the results of these additional tests, either both ranges are discarded or only one. A median method is then applied to the remaining ranges:

$>$ Select 14 ranges randomly (depends on the noise and on the number of ranges),

$>\quad$ Compute the solution to equation (3),

$>$ Characterize the solution with the median of the range residuals.

Repeat these steps $\mathrm{N}$ times and keep the set of 14 ranges providing the smallest median residual. $\mathrm{N}$ has to 
be chosen even larger that the range measurements are noisy. This is of course at the expense of a longer computation time. For the simulations, we used $\mathrm{N}=70$, the ranges noises is: $20 \%$ spurious and $20 \%$ affected by a Gaussian noise with a $10 \mathrm{~m}$ standard deviation.

\section{Uncertainty on the initial estimation}

The system of equations (3) can be expressed by:

$$
d=f(X, \psi, \theta, u, \Delta t)+\xi
$$

Where $\mathrm{d}$ is the vector of the selected ranges, $\mathrm{X}=\left(\mathrm{x}, \mathrm{y}, \mathrm{v}_{\mathrm{cn}}, \mathrm{v}_{\mathrm{ce}}, \mathrm{du}\right)^{\mathrm{t}}$, and $\xi$ is a Gaussian noise on the measured ranges with variance matrix $\mathrm{R}$. The non-linear least squares optimization provides the covariance matrix of X (Cramer-Rao lower bound):

$$
P=\left[\left(\frac{\partial f(X)}{\partial X}\right)^{t}\left(H P_{e} H_{t}+R\right)^{-1}\left(\frac{\partial f(X)}{\partial X}\right)\right]^{-1}
$$

Where $H$ is the Jacobian of the ranges with respect to $\psi, \theta, \mathrm{u}$ and $\mathrm{P}_{\mathrm{e}}$ is the covariance matrix of $\psi, \theta, \mathrm{u}$. The result of the optimization is used to initialize the state vector of the Kalman filter described below, and the covariance matrix $\mathrm{P}$ is used to initialize the estimation error covariance matrix of the filter.

\section{EXTENDED KALMAN FILTER}

\section{A. State equation}

The state vector is made up of the vehicle's position $(\mathrm{x}, \mathrm{y})$ in $\mathrm{R}_{0}$, the vehicle's depth $(\mathrm{z})$, the components of the underwater current $\left(\mathrm{v}_{\mathrm{cn}}, \mathrm{v}_{\mathrm{ce}}\right)$ and the speed bias $(\mathrm{du})$. The inputs are the vehicle's heading $\psi$, its pitch $\theta$ and its water-referenced speed $u$. The covariance matrix of the inputs is the diagonal matrix $C_{i n}=\operatorname{diag}\left(\sigma_{\psi}^{2}, \sigma_{\theta}^{2}, \sigma_{u}^{2}\right)$. The current and the speed bias are modeled as constant. The state noise vector isv $\mathrm{k}_{\mathrm{k}}$ and its covariance matrix $\mathrm{Q}_{\mathrm{k}}$. The state equation is then expressed by:

$$
\left[\begin{array}{c}
x \\
y \\
z \\
v_{c n} \\
v_{c e} \\
d u
\end{array}\right]_{k+1}=\left[\begin{array}{c}
x \\
y \\
z \\
v_{c n} \\
v_{c e} \\
d u
\end{array}\right]_{k}+\left[\begin{array}{c}
C \theta C \psi(u-d u)+v_{c n} \\
C \theta S \psi(u-d u)+v_{c e} \\
-S \theta(u-d u) \\
0 \\
0 \\
0
\end{array}\right]_{k} \Delta t+v_{k}
$$

\section{B. Observation equation}

The observation equation expresses the acoustic round-trip time of flight $(\mathrm{T})$ as a function of the vehicle's state at the time of the ping and at the time the vehicle receives the reply from the beacon. The vehicle's motion between the ping and the reception is then taken into account, and observations are expressed in terms of all the state variables. The measurement noise on the travel times is represented by $\mathrm{w}_{\mathrm{k}}$. It variance is $R_{k}$. The speed of sound is noted $c$ and the beacon turn around time $\Delta \mathrm{t}_{\mathrm{r}}$.

$$
\begin{aligned}
& T=\frac{1}{c}\left[\left(x_{b}-x_{\text {ping }}\right)^{2}+\left(y_{b}-y_{\text {ping }}\right)^{2}+\left(z b-z_{\text {ping }}\right)^{2}\right]^{1}+ \\
& \left.\left[\left(x_{b}-x_{\text {recept }}\right)^{2}+\left(y_{b}-y_{\text {recept }}\right)^{2}+\left(z b-z_{\text {recept }}\right)^{2}\right]^{2}\right\} \Delta t_{r}+w_{k}
\end{aligned}
$$

In general, a time of flight is buffered by the acoustic ranging system and made available for processing after a preset timeout following the ping time. The vehicle's estimated state at that time is $\mathrm{X}_{\mathrm{k} / \mathrm{k}}$. The prediction step being applied first, the vehicle predicted state is $X_{k+1 / k}$. The ping time $\left(t_{\text {ping }}\right)$ is known and the reception time $\left(t_{\text {recept }}\right)$ can be calculated by adding the time of flight to the ping time.

Since the state prediction does not modify the current components and the speed bias estimates, these estimates are constant from the ping time to the time of flight processing time. The vehicle's position is then back propagated in time using the current state and the inputs, which were memorized since the ping.

\section{Equations of the filter}

The filter equations are different from the classical equations in that inputs $\mathrm{U}_{\mathrm{k}}=(\psi, \theta, \mathrm{u})^{\mathrm{t}}$ can be found both in the state and the observation equations. Equations 6) and (7) can be rewritten:

$$
\left\{\begin{array}{l}
X_{k+1}=f\left(X_{k}, U_{k}\right)+V_{k} \\
Z_{k}=h\left(X_{k}, U_{k}\right)+W_{k}
\end{array}\right.
$$

The filter proceeds in two steps. The first step is a prediction of the state based on the inputs in $\mathrm{U}_{\mathrm{k}}$. It basically corresponds to dead reckoning using the last estimates of the underwater current. The state is predicted together with its prediction error covariance $\mathrm{P}_{\mathrm{k}+1 / \mathrm{k}}$ :

$$
\left\{\begin{array}{l}
X_{k+1 / k}=f\left(X_{k / k}, U_{k}\right) \\
P_{k+1 / k}=F_{k} P_{k / k} F_{k}^{t}+J_{k} C_{i n} J_{k}^{t}+Q_{k}
\end{array}\right.
$$

The second step takes place when a time of flight is available. The predicted state is corrected based on the information carried by the new measurement. The prediction error covariance matrix $\mathrm{P}_{\mathrm{k}+1 / \mathrm{k}+1}$ is also computed:

$$
\left\{\begin{array}{l}
X_{k+1 / k+1}=X_{k+1 / k}+K_{k+1}\left(Z_{k+1}-h\left(X_{k+1 / k}, U_{k}\right)\right) \\
P_{k+1 / k+1}=P_{k+1 / k}-K_{k+1}\left(H_{k+1} P_{k+1 / k}+S_{k+1}^{t}\right)
\end{array}\right.
$$

The following Jacobian matrices have to be calculated as usually.

$\mathrm{S}_{\mathrm{k}+1}=\mathrm{J}_{\mathrm{k}+1} \mathrm{C}_{\mathrm{in}} \mathrm{D}_{\mathrm{k}+1}^{\mathrm{t}}$ is a correlation term accounting for the fact that inputs are both in the state and the observation equations. Because of this correlation, the expression of the Kalman gain is a little more complicated than usual: 


$$
\begin{aligned}
& \mathrm{K}_{\mathrm{k}+1}=\left(\mathrm{P}_{\mathrm{k}+1 / \mathrm{k}} \mathrm{H}_{\mathrm{k}+1}^{\mathrm{t}}+\mathrm{S}_{\mathrm{k}+1}\right) \\
& \left(\mathrm{H}_{\mathrm{k}+1} \mathrm{P}_{\mathrm{k}+1 / \mathrm{k}} \mathrm{H}_{\mathrm{k}+1}^{\mathrm{t}}+\mathrm{D}_{\mathrm{k}+1} \mathrm{C}_{\mathrm{in}} \mathrm{D}_{\mathrm{k}+1}^{\mathrm{t}}+\mathrm{R}+\mathrm{H}_{\mathrm{k}+1} \mathrm{~S}_{\mathrm{k}+1}+\mathrm{S}_{\mathrm{k}+1}^{\mathrm{t}} \mathrm{H}_{\mathrm{k}+1}^{\mathrm{t}}\right)^{-1}
\end{aligned}
$$

\section{Time of flight validation}

When a time of flight is available, its validity is first checked by means of the Mahalanobis distance. The innovation and its variance are calculated by:

$$
\begin{aligned}
1_{k+1} & =T_{k+1}-h\left(X_{k+1 / k}, U_{k+1}\right) \\
P_{i_{k+1}} & =H_{k+1} P_{k+1 / k} H_{k+1}^{t}+D_{k+1} C_{i n} D_{k+1}^{t}+R \\
& +H_{k+1} S_{k+1}+S_{k+1}^{t} H_{k+1}^{t}
\end{aligned}
$$

The time of flight $T_{k+1}$ is validated and used to correct the predicted state if it passes the following test:

$$
\mathrm{i}^{\mathrm{t}} \text { with } \gamma^{2} \text { typically equal to } 1.32 \text {. }
$$

\section{VEHICLE NAVIGATION}

A study of the information matrix similar to that described in [6] has showed that the trajectory that maximizes the information (or conversely minimize the estimation error covariance) consists of leaving the beacon on either side of the vehicle so that the vehicle's heading deviates by $90^{\circ}$ from the line joining the vehicle and the beacon (the vehicle describes a circle centered at the beacon). In order to increase the information, we use the following procedure. At the end of the $360^{\circ}$ rotation, the vehicle is commanded to move at $90^{\circ}$ with respect to the beacon. When this condition is achieved, the volume of the prediction error covariance ellipsoid is calculated by:

$\mathrm{C}_{0}=\sqrt{\operatorname{det}(\mathrm{P})}$

When a new time of flight is made available and used by the filter, the volume is calculated for the new covariance matrix:

$$
\mathrm{C}_{\mathrm{k}}=\sqrt{\operatorname{det}\left(\mathrm{P}_{\mathrm{k} / \mathrm{k}}\right)}
$$

The homing is not satisfying since the vehicle never reaches the beacon. Going straight to the beacon is, however, neither a desired configuration because the range measurements cannot correct efficiently lateral errors along the vehicle track, so that the vehicle could actually miss the beacon. In the case of speed bias is considered, the heading command law described in [6] do not satisfied the speed convergence even if we change the speed of the vehicle during homing. We notice that the deviation arrived quickly to $56^{\circ}$. We introduced the distance to the beacon ("range") in the deviation angle:

$$
\varnothing_{\mathrm{k}}=\varnothing_{\mathrm{b}_{\mathrm{k}}}+\frac{\partial}{2}\left[1-\exp \left(k * \text { range } * \frac{\mathrm{C}_{\mathrm{k}}}{\mathrm{C}_{k}-\mathrm{C}_{0}}\right)\right]
$$

Where $\mathrm{k}$ is a speed homing coefficient.
It's very difficult for the filter to converge to the real speed bias when heading is changing all the time (the speed bias is considered on the longitudinal vehicle axis). For this reason, we keep the same direction during some measurements before compute the new heading.

The case of navigation is simplest, the vehicle keeps circling around the beacon until the ration of $\mathrm{C}_{\mathrm{k}}$ over $\mathrm{C}_{0}$ is smaller than a preset threshold (function of $\mathrm{N}$ ). The AUV then reaches its first waypoint and starts doing its survey. Then, the vehicle still uses the filter to estimate its position during the survey.

\section{MULTIPLE VEHICLE OPERATIONS}

The principle is almost the same than the method presented above. We extend this technique to a mobile beacon which is assimilated to a leader vehicle. We suppose that the second vehicle knows occasionally (by way of an acoustic modem) the dead-reckoned leader displacement. This slave vehicle initialize the leader location by commanding a $360^{\circ}$ rotation to himself while ranging to the leader at a regular rate and measuring its own displacements between pings. For a better following and a small distancing of vehicles during the initialization phase, the slave vehicle trajectory can be two semi circle.

The slave can know the leader displacement by dead-reckoning and compute his location like:

$\left[\begin{array}{c}x_{b} \\ y_{b} \\ z_{b}\end{array}\right]_{k+1}=\left[\begin{array}{c}x_{b} \\ y_{b} \\ z_{b}\end{array}\right]_{k}+\left[\begin{array}{c}\operatorname{Cos} \psi_{b} \operatorname{Cos} \theta_{b} U_{b} \\ \operatorname{Sin} \psi_{b} \operatorname{Cos} \theta_{b} U_{b} \\ -\operatorname{Sin} \theta_{b} U_{b}\end{array}\right] \Delta t$

where $\left(\psi_{\mathrm{b}}, \theta_{\mathrm{b}}, \mathrm{U}_{\mathrm{b}}\right)$ are transmitted by acoustic link and $\left(\mathrm{x}_{\mathrm{b}}, \mathrm{y}_{\mathrm{b}}, \mathrm{z}_{\mathrm{b}}\right)_{0}=(0,0,0)$.

We suppose that the leader knows with precision his position (he is not affected by the underwater current and he knows his absolute speed). In such a situation, only the slave dead reckoned displacements can be affected by errors due to underwater currents.

\section{SIMULATION RESULTS}

Our search group has developed a hydrodynamic simulator for Taipan vehicle. It allows us to simulate the behavior of this underwater vehicle by considering a lot of parameters. For example, it does not only take into account the applied command, but also the environmental parameters and hydrodynamic constants of the vehicle. This simulator runs on PC computers and has been developed under Matlab ${ }^{\mathrm{TM}}$ environment. Its validity has been proved thanks to many comparisons with real data collected during experimentations of Taipan in lagoons and open sea. The algorithm described above has been tested in simulation (where sensors simulations give data affected by a Gaussian noise with an $0.5 \mathrm{~m}$ standard deviation) and in postprocessing experimentation. 
In the homing simulation (Fig.3), the current was set so as to have a $0.2 \mathrm{~m} / \mathrm{s}$ magnitude and a $60^{\circ}$ direction. The speed bias was set at $0.2 \mathrm{~m} / \mathrm{s}$. In order to simplify the visualization of the tracks, the absolute position of the beacon is subtracted from all the absolute positions. The beacon then appears to be located at $(0,0)$ on the plots. During the initialization phase, the vehicle deadreckons its displacements between ranges without any knowledge of its absolute initial position. For the plots, however, the dead-reckoned position was initialized by the vehicle's actual initial position. After the initialization, the filter provides the estimated position of the vehicle in $R_{0}$, which is used to steer the AUV so as to leave the beacon at 90 . When you realize homing, the difficulty is to reduce the distance without deteriorate filter solutions. In figure 3, we can see the difficult speed bias convergence. This convergence is more precise when you bring nearer to the beacon. We think that this is the fact that dead-reckoned and distance are in the same order.
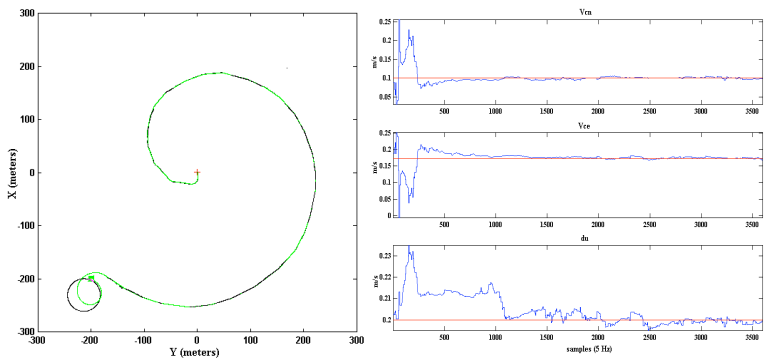

Fig.2 : homing trajectory (dark: estimated, light: actual) and perturbations results

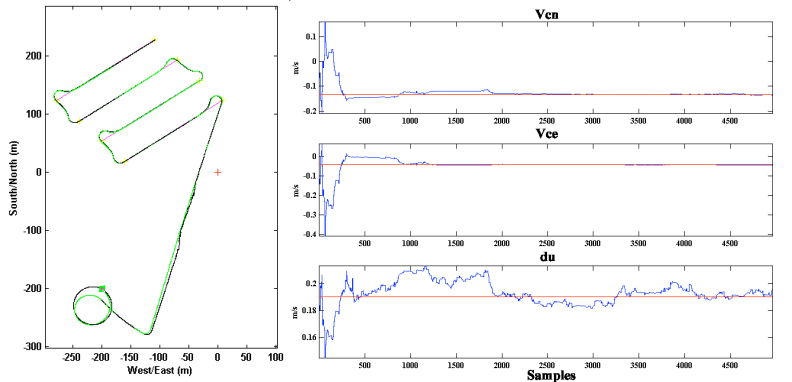

Fig.3 : Parallel trajectory (dark: estimated, light: actual) and perturbations results

We also present results for two different vehicle tracks. The first track consists of parallel legs (Fig.4) with a current of $0.14 \mathrm{~m} / \mathrm{s}$ magnitude and $197^{\circ}$ direction, the speed bias is $0.19 \mathrm{~m} / \mathrm{s}$. The second track consists of radial legs (Fig.5) with a current of $0.11 \mathrm{~m} / \mathrm{s}$ magnitude and $72^{\circ}$ direction, the speed bias is $0.15 \mathrm{~m} / \mathrm{s}$. The AUV starts its $360^{\circ}$ rotation at the square mark located at about $(-200,-200)$ with an initial heading of about $135^{\circ}$. The dead-reckoned path is a circle (dark line) since the vehicle is not aware of the current. The actual vehicle trajectory is show by the distorted circle (light line). The estimated position of the vehicle at the end of the initialization is shown by a cross at about $(-200,-150)$ in figure 4. It can be seen that this estimated position is very close to the actual position of the vehicle after the distorted circle.

The estimated position then jumps from the end of the circle to these coordinates. From there, the actual and estimated trajectories are very close to each other and remain so until the end. It can be seen that the perturbations estimations converge to the correct values during the $90^{\circ}$ navigation phase. The radial track gives better results principally because of the vehicle travels at varying headings and during different straight line.
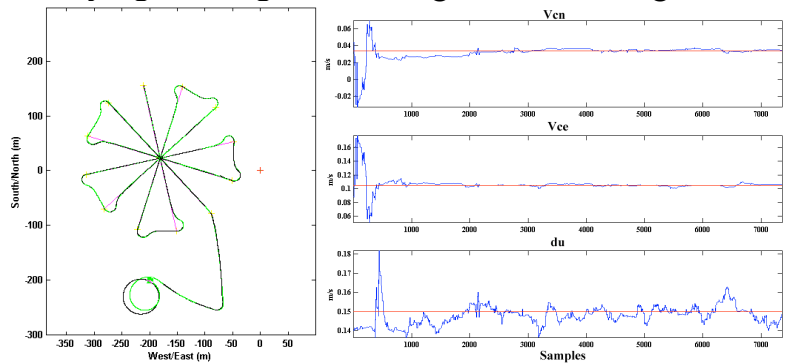

Fig.4 : Radial trajectory (dark: estimated, light: actual) and perturbations results

\section{POST PROCESSING COMPARISON}

In GOATS 2000 campaign (NATO experimentation), th Odyssey AUV used LBL positioning for his navigation. We used some of it experiments for a comparison with our method. LBL navigation is realized with respect to 5 beacons scattering in Biodola bay (Elba island). For our postprocessing computation, we consider beacons which AUV distances seems to be as perpendicular as possible with the AUV navigation. The vehicle ping each 10s, for this reason, we could not consider the initialization phase. We initialized the filter with a GPS position.

In Fig 5 and Fig 6, we show post-processing comparison. At the left, we show at the top navigation with respect to one beacon and at the bottom, LBL navigation. At the right, we show at the top $\mathrm{x}$ and $\mathrm{y}$ differences between these methods and at the bottom, perturbations estimations.

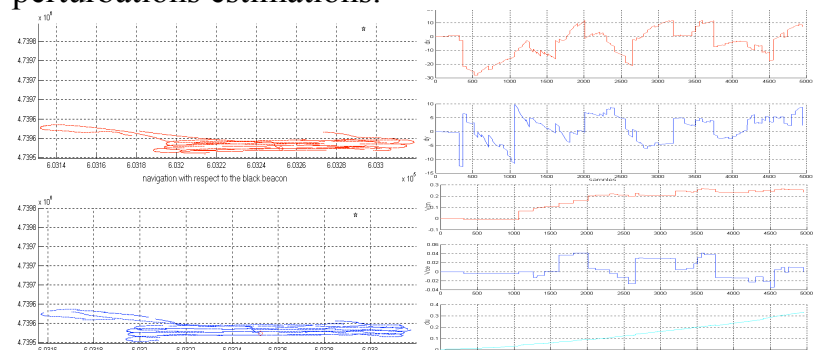

Fig 5 : first comparison and perturbations estimations between LBL and our method

We could say in the two comparisons that differences between LBL and our method are converge to zero. We estimate some underwater current and a speed bias but there is an other perturbation which is 
include in the other. We had seen during experimentation that the AUV had a heading bias and don't estimate it. For that, we can't validate perturbations results. These extremes conditions shows the robustness of the filter because of we don't do anything that we wanted to do in simulation and it's working.

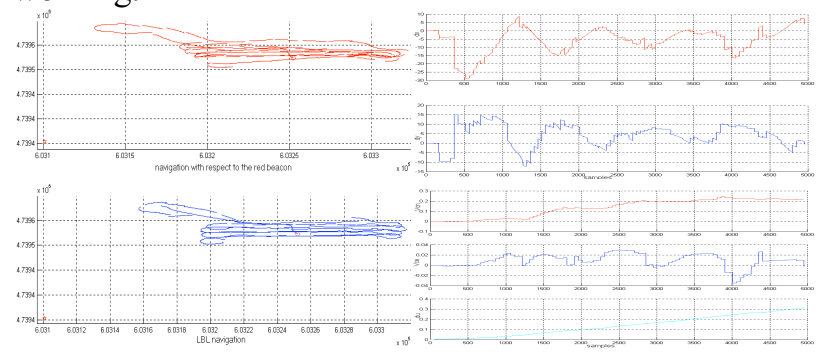

Fig 6 : second comparison and perturbations estimations between LBL and our method

\section{POST PROCESSING EXPERIMENTATION}

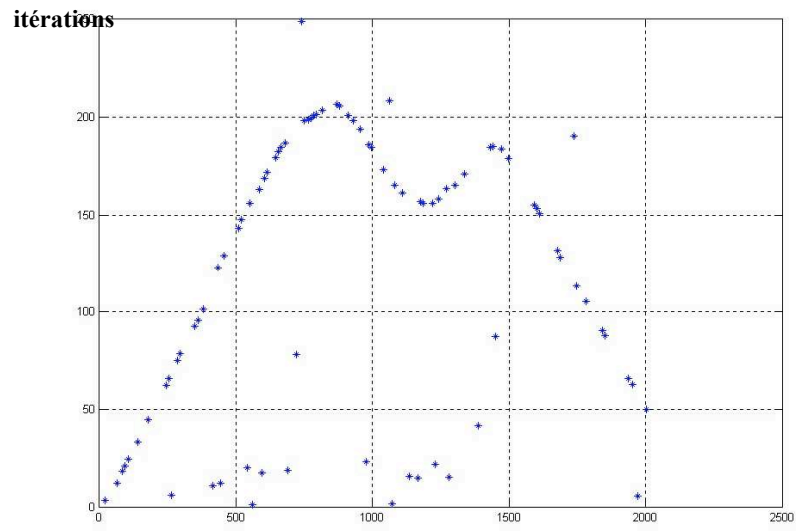

Fig 7 : ranges between the vehicle ans the beacon during all the mission

Before realize real experimentation with TAIPAN AUV, we would like tested our method with real data of this vehicle. Experimentation would be realized near "Vise" thermal spring in "Thau" pond.

The vehicle is navigating only with his own dead reckoned. So, the vehicle is affected by a biais due to speed vs. rpm calibration errors and by the unknown underwater current component. We use a fixed station in wicht an acoustic modem is cooperating with the vehicle for measuring the distance. The Fig 7 represents real ranges between the beacon and the vehicle. The vehicle's trajectories represented in Fig 8. We don't have an absolute positioning system for compare the estimated final point and the real value. Just, we could say that visually, the arrival zone by Kalman filtering is correct as compare as final dead-reckoned position. We made verification on the vehicle and we confirm that he has a $0.16 \mathrm{~m} / \mathrm{s}$ speed biais.
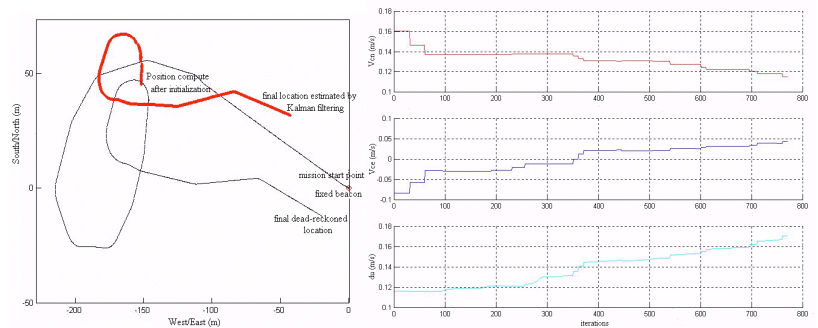

Fig 8 : dead-reckoned vehicle trajectory and post processing using one beacon results

\section{MULTIPLE VEHICLE NAVIGATION}

We show in different missions the utility and the performance of our method. The both vehicles are at different depths. Time of flight measurements are acquired each two seconds. The current was set so as to have a $0.2 \mathrm{~m} / \mathrm{s}$ magnitude and a randomly direction.

During the initialization phase, the slave deadreckons without any knowledge of its initial position with respect to the leader. After the initialization, the filter directly provides the estimated position of the vehicle in $R_{b}$ frame (define at the end of slave rotation. The values $\mathrm{dx}$ and dy represent respectively north and east errors locations for the slave vehicle.

\section{A. "Zig-zag" trajectory following}
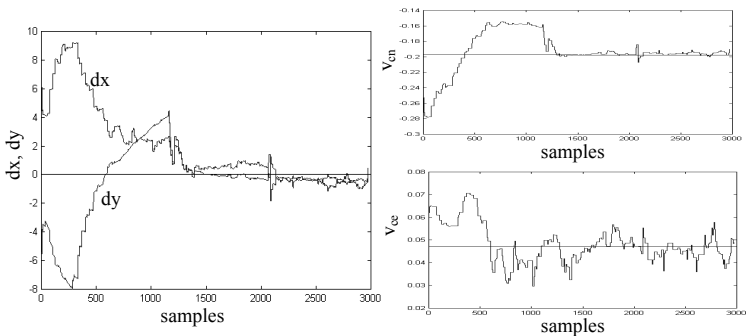

Fig. 9 : results of the navigation

In this mission, the leader must navigate along a "zig-zag" trajectory (Fig 10), the other vehicle must join its location.

Changing leader direction modifies the follower trajectory (which looks like a circle). This entails the convergence of the algorithm (Fig 9).

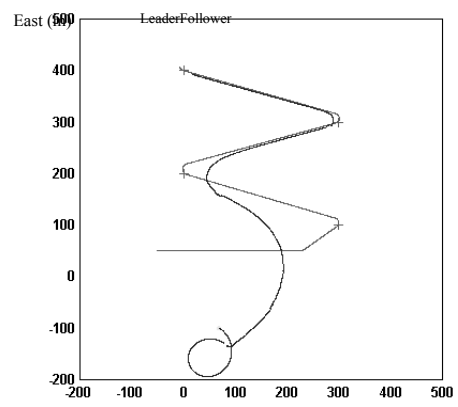

Fig. 10 : Estimated AUVs trajectory 


\section{B. pipeline following}

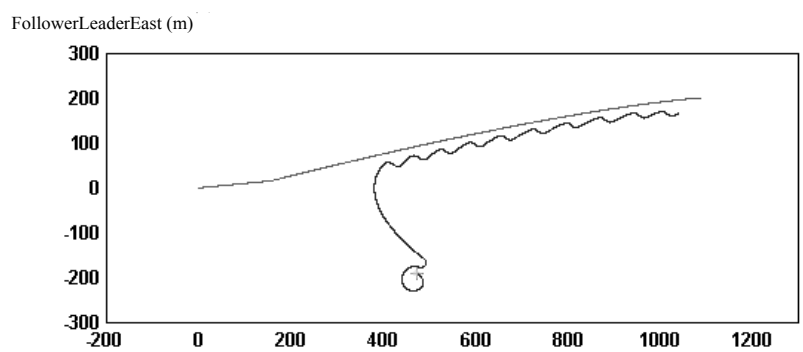

Fig. 11 : pipeline following mission

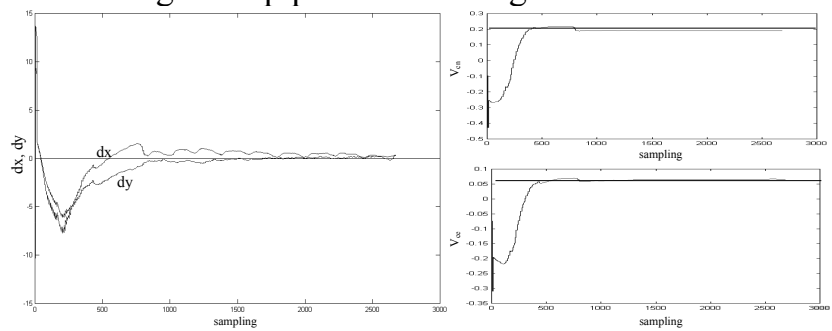

Fig. 12 : filter results

In this example, we assign the leader vehicle to follow a pipeline (Fig. 11). The follower must position the leader and transmit its absolute position. It is a subsurface vehicle.

To accelerate the filter convergence (Fig. 12), the follower change his yaw when it has the same that the leader. The covariance matrix evolution provide us information about the filter convergence. When convergence has been reached, the slave vehicle can follow the leader direction without any heading change.

\section{C. scanning area}

The scanning of an area is important for exploration and for data recovering for post processing. We show here the utility of this kind of mission which allows data measurements in the same space time (Fig. 13). It appears that an efficient exploration task requires firstly a correct convergence of the positioning algorithm induced by a correct initialization step (Fig. 14).

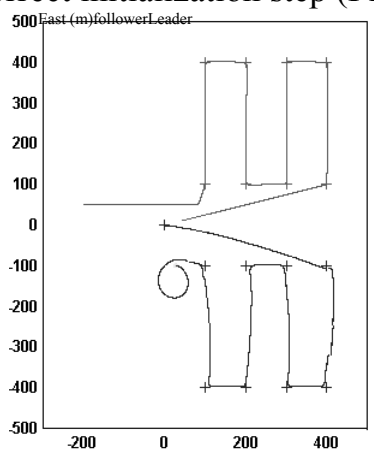

Fig. 13 : scanning mission

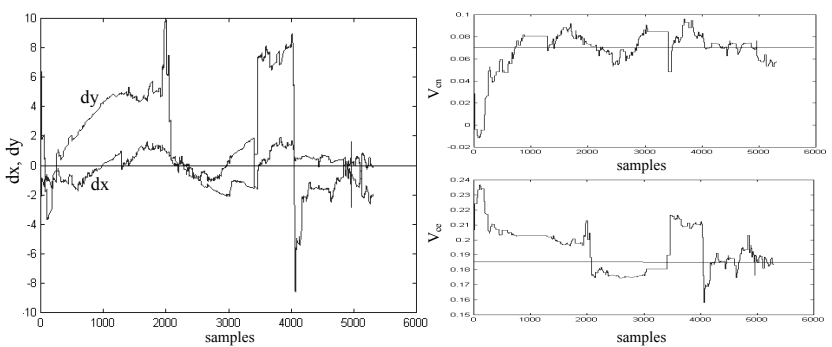

Fig. 14 : Convergence results

\section{CONCLUSION}

In conclusion, we can say that this navigation method is robust and efficient.

In addition to its interest as an alternate solution to classical long baseline, we believe that the range-only solution could be experiment for multiple AUV operation. For that, the slave needs to modify its heading during the beginning of the navigation. Then, after the convergence, yaw can follow a constant direction. A second reason of the convergence is the precision of the initialization phase.

Our future works concerns multiple vehicles experimentation, movement generation and docking.

\section{REFERENCES}

[1] J.G. Bellingham, T.R. Consi, U. Tedrow and D. Di Massa, "Hyperbolic acoustic navigation for underwater vehicles: implementation and demonstration", Proceedings of the 1992 symposium on Autonomous Underwater Vehicle Technology, June 2-3, Washington, DC, USA.

[2] D.K. Atwood, J.J. Leonard, J.G. Bellingham, and B. A. Moran, "An acoustic navigation system for multiple vehicles", 9th Int. Symp. on Unmanned Untethered Submersible Technology, pp.202-208, New Hampshire, September 25-27, 1995.

[3] J. Vaganay, J.G. Bellingham and J.J. Leonard, "Comparison of fix computation and filtering for autonomous acoustic navigation", International Journal of Systems Science, vol.29, nº10, pp. 1111-1122, 1998.

[4] A. Matos, N. Cruz, A. Martins and F.L. Pereira, "Development and implementation of low-cost LBL navigation system for an AUV', OCEANS '99 MTS/IEEE. Riding the Crest into the $21^{\text {st }}$ Century, volume 2, pages 774779.

[5] A.P. Scherbatyuk, "The AUV positioning using ranges from one transponder LBL", OCEANS '95 MTS/IEEE. Challenges of Our changing Global Environment. Conference Proceedings., Volume 3, pages 1620-1623.

[6] J.Vaganay, P. Baccou, B. Jouvencel, "Homing by acoustic ranging to a single beacon", OCEANS '00 MTS/IEEE, Conference and Exhibition, Proceedings Volume 2, pages 1457-1462, September 11-14, Providence, RI, 2000.

[7] M.B. Larsen, "Synthetic long baseline navigation of underwater vehicles", OCEANS '00 MTS/IEEE, Conference and Exhibition, Proceedings Volume 3, pages 2043-2050, September 11-14, Providence, RI, 2000.

[8] P. Baccou, B. Jouvencel, V. Creuze, "Single beacon acoustic for AUV navigation" International Conference on Advanced Robotics, Budapest Hungary, 22-25 August, 2001. 